\title{
Nonmonotonic Classical Magnetoconductivity of a Two-Dimensional Electron Gas in a Disordered Array of Obstacles
}

\author{
N. H. Siboni, ${ }^{1}$ J. Schluck, ${ }^{2}$ K. Pierz, ${ }^{3}$ H. W. Schumacher, ${ }^{3}$ D. Kazazis, ${ }^{4, \$}$ J. Horbach, ${ }^{1,{ }^{*}}$ and T. Heinzel ${ }^{2, \dagger}$ \\ ${ }^{1}$ Institut für Theoretische Physik II, Heinrich-Heine-Universität, Universitätsstraße 1, 40225 Düsseldorf, Germany \\ ${ }^{2}$ Institut für Experimentelle Physik der kondensierten Materie, Heinrich-Heine-Universität, \\ Universitätsstraße 1, 40225 Düsseldorf, Germany \\ ${ }^{3}$ Physikalisch-Technische Bundesanstalt, Bundesallee 100, 38116 Braunschweig, Germany \\ ${ }^{4}$ CNRS, Université Paris-Sud, Université Paris-Saclay, C2N Marcoussis, 91460 Marcoussis, France
}

(Received 2 August 2017; published 2 February 2018)

\begin{abstract}
Magnetotransport measurements in combination with molecular dynamics simulations on twodimensional disordered Lorentz gases in the classical regime are reported. In quantitative agreement between experiment and simulation, the magnetoconductivity displays a pronounced peak as a function of the perpendicular magnetic field $B$ which cannot be explained by existing kinetic theories. This peak is linked to the onset of a directed motion of the electrons along the contour of the disordered obstacle matrix when the cyclotron radius becomes smaller than the size of the obstacles. This directed motion leads to transient superdiffusive motion and strong scaling corrections in the vicinity of the insulator-to-conductor transitions of the Lorentz gas.
\end{abstract}

DOI: 10.1103/PhysRevLett.120.056601

A system of noninteracting particles moving in a Poisson-distributed array of identical obstacles is known as a Lorentz gas. Originally proposed for the motion of electrons in a metal [1], the Lorentz gas has developed into a universal model for transport phenomena in many types of heterogeneous media, like anomalous diffusion in colloidal and biosystems [2-10], microwave-induced magnetoresistance oscillations [11], or negative magnetoresistance in metallic and semiconductor systems [12-17]. Versatile implementations of Lorentz gases can be realized experimentally by two-dimensional electron gases (2DEGs) exposed to a random array of obstacles. Such systems provide a high intrinsic electron mobility and the option to pattern the obstacles lithographically. A perpendicular magnetic field $B$ tunes the cyclotron radius $R_{\text {cy }} \propto B^{-1}$ of the electronic motion, acting as an additional characteristic length scale.

Experimental studies of the magnetotransport of 2DEGs in disordered obstacle arrays have been scarce (see, e.g., Refs. [18-23]). Especially, the magnetoconductivity $\sigma_{x x}(B)$ in a regime of high obstacle densities $n^{\star}$ and large magnetic fields has not been systematically explored up to now. Here, $n^{\star}$ denotes the dimensionless number density of obstacles, $n^{\star}=(N / A) R_{\text {int }}^{2}$, with $N$ the number of obstacles, $A$ the area of the system, and $R_{\text {int }}$ the interaction distance between an electron and an obstacle, i.e., the effective radius of the (circular) obstacles. In this Letter, we present a classical experimental realization of a Lorentz gas in combination with classical molecular dynamics (MD) simulations and demonstrate that the electron transport qualitatively changes if the cyclotron radius $R_{\text {cy }}$ becomes smaller than the interaction distance $R_{\text {int }}$. As expected from kinetic theories, the conductivity $\sigma_{x x}(B)$ is a monotonically decaying function at low densities. For large densities and $R_{\text {cy }} \lesssim R_{\text {int }}$, however, it exhibits a maximum that moves to larger values of $B$ with increasing $n^{\star}$. This maximum has been observed in simulations [24,25], but it has hitherto remained unexplained and not been observed experimentally. Our study provides the first experimental verification of the phase diagram for the magnetotransport in the 2D Lorentz gas, and our simulation analysis elucidates a so far unexplored dynamic regime.

The magnetotransport in the 2D Lorentz gas is associated with two insulator-to-conductor transitions at high and low obstacle density $n^{\star}$ which are due to underlying static percolation transitions $[24,25]$. The location of the transition at a high density is independent of the magnetic field $B$ and located at a critical density $n_{c}^{\star}=0.359$ for a Poissondistributed arrangement of overlapping disks: While for $n<n_{c}^{\star}$ the electron exhibits diffusive transport through the void space between the obstacles, for $n>n_{c}^{\star}$ the void space is disconnected into finite pockets in which the electron is trapped. The second, $B$-dependent localization transition occurs at a density $n_{\mathrm{ld}, c}^{\star}(B)<n_{c}^{\star}$. It can be understood in terms of skipping orbits that the electrons, acting as tracer particles in this experimental implementation, perform around the obstacles, or clusters thereof. This localizes all particles as $n^{\star}$, or the cyclotron radius $R_{\text {cy }}$, respectively, is decreased [24].

For a fixed $B$ field, the magnetoconductivity exhibits a maximum as a function of $n^{\star}$ which is located at $n_{\mathrm{ld}, c}^{\star}<n_{\max }^{\star}<n_{c}^{\star}$. This maximum is intimately related to 
the maximum in $\sigma_{x x}(B)$. The line of maxima, $n_{\max }^{\star}(B)$, is the geometric mean of the two critical densities. The change of the transport for $R_{\mathrm{cy}} \lesssim R_{\text {int }}$ along the line $n_{\max }^{\star}(B)$ is due to a change of the motion of the tracer particles (electrons) from a diffuse scattering by the obstacles to a directed motion along the contour of the obstacle arrangement. In the limit $B \rightarrow \infty$, this directed motion completely dominates the transport and suppresses the critical slowing down at $n_{\mathrm{ld}, c}^{\star}$ and $n_{c}^{\star}$. At finite but high $B$ fields, it leads to strong corrections to the scaling behavior in the vicinity of the critical points.

A $\mathrm{GaAs} / \mathrm{Al}_{0.3} \mathrm{Ga}_{0.7} \mathrm{As}$ heterostructure with a $2 \mathrm{DEG}$ located $150 \mathrm{~nm}$ below the surface is used. The electron density and mobility are $n_{e}=2.5 \times 10^{15} \mathrm{~m}^{-2}$ and $340 \mathrm{~m}^{2} / \mathrm{V} \mathrm{s}$, respectively, corresponding to a mean free path of $31 \mu \mathrm{m}$ at temperatures below $1 \mathrm{~K}$. The repulsive Lorentz obstacles are formed by circular holes in the 2DEG. They are patterned by electron beam lithography and subsequent reactive ion etching. Within each array, the obstacles are of identical size, while their positions are Poisson distributed, with mutual overlaps allowed. All disks have a lithographic radius of $425 \mathrm{~nm}$; see Fig. 1(a). From Aharonov-Bohm measurements in large magnetic fields [26], we estimate [27] the lateral depletion length to $\approx 75 \mathrm{~nm}$, such that the effective electronic disk radius is $R_{\mathrm{int}} \approx 500 \mathrm{~nm}$. The chip contains
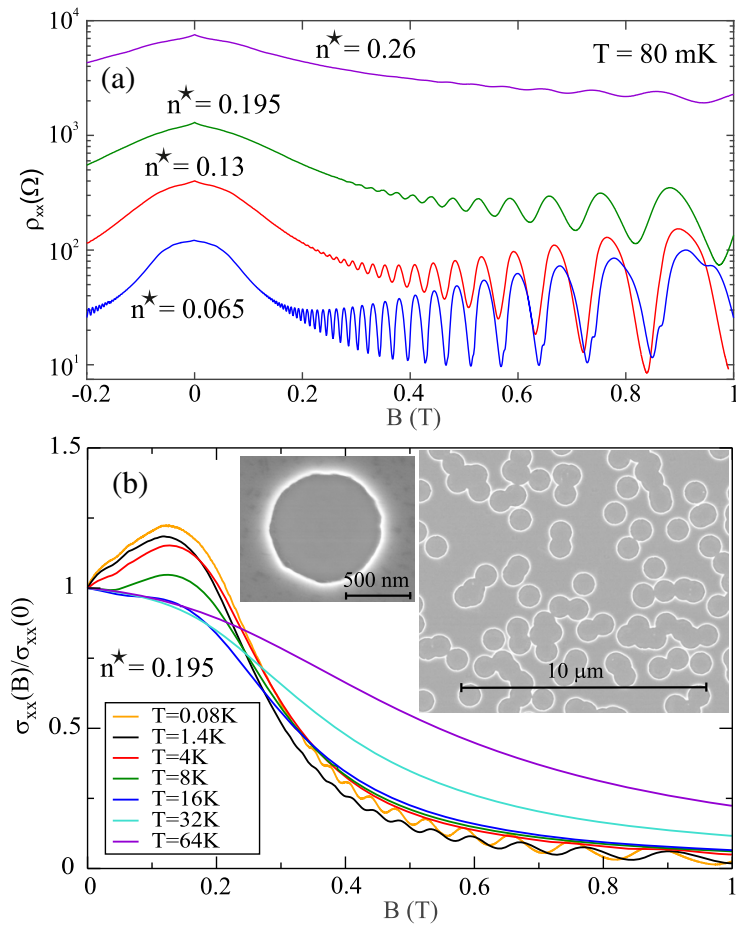

FIG. 1. (a) Longitudinal magnetoresistivities $\rho_{x x}$ for various obstacle densities $n^{\star}$, measured at a temperature of $\approx 80 \mathrm{mK}$. (b) The magnetoconductivity $\sigma_{x x}(B)$ and its temperature dependence for $n^{\star}=0.195$. Insets: Scanning electron microscope picture of a Lorentz array section with $n^{\star}=0.195$ and magnified view of a single disk. four Lorentz arrays with disk densities $n^{\star}=0.065,0.13$, 0.195 , and 0.26 . The arrays have an area of $200 \mu \mathrm{m}$ by $100 \mu \mathrm{m}$. The mean free path due to the scattering at the disks is $4.0,2.0,1.3$, and $1.0 \mu \mathrm{m}$, respectively.

The samples were measured in a dilution refrigerator with a base temperature of $8 \mathrm{mK}$. The electron temperature is estimated to $\approx 80 \mathrm{mK}$. A ${ }^{4} \mathrm{He}$ gas flow cryostat with a variable temperature insert and a base temperature of $1.4 \mathrm{~K}$ is used for measurements at temperatures above $1 \mathrm{~K}$. An ac current $(500 \mathrm{nA}, 17.7 \mathrm{~Hz})$ is injected. The longitudinal and Hall voltages are measured using lock-in amplifiers.

The longitudinal magnetoresistivity $\rho_{x x}(B)$ [see Fig. 1(a)] shows a strong peak around $B=0$ which in some arrays extends well into the range where Shubnikov-de Haas oscillations [28] are observed. As $n^{\star}$ is increased from 0.065 to $0.26, \rho_{x x}(0)$ increases by approximately a factor of 50. In addition, a small, narrow peak on top at $B=0$ is observed which will be discussed elsewhere.

The longitudinal magnetoconductivity is obtained from the measured resistivity components via $\sigma_{x x}(B)=\rho_{x x}(B) /$ $\left[\rho_{x x}^{2}(B)+\rho_{x y}^{2}(B)\right]$, where $\rho_{x y}(B)$ denotes the Hall resistivity (see Supplemental Material [29]). In Fig. 1(b), the obtained $\sigma_{x x}(B)$ is shown for the array with $n^{\star}=0.195$ for various temperatures. A pronounced maximum at $B \approx$ $140 \mathrm{mT}$ is observed. It shows a weak temperature dependence and evolves at higher temperatures into a shoulder that is still visible at $32 \mathrm{~K}$. This weak temperature dependence indicates a classical origin. We have observed the same phenomenology in a set of scaled samples with identical number densities but with $R_{\text {int }}=1 \mu \mathrm{m}$ [29]. This behavior is in qualitative contradiction to both the Boltzmann model as well as to the Bobylev model valid for Lorentz gases with small $n^{\star}$ [30]. Rather, it is associated with the abovementioned conductivity maximum as predicted by numerical simulations for high-density Lorentz gases [24,25]. A magnetoconductivity maximum in $2 \mathrm{D}$ arrays of randomly placed obstacles is actually visible in the data reported by Lütjering [20]. It has, however, a different phenomenology than the data discussed here and is probably related to the anomalous weak localization present in these samples [23]. We note that, in arrays of periodic obstacles, magnetoconductivity peaks are well known; see, e.g., Refs. [31,32]. They have their origin in electron trajectories commensurate with the lattice [33] and are thus of a distinctly different character.

MD simulations of a system of noninteracting fluid particles in a $2 \mathrm{D}$ matrix of randomly placed obstacle particles are performed using LAMMPS [34]. Matrix (index $M)$ and fluid particles $(F)$ interact via a shifted, purely repulsive Weeks-Chandler-Andersen potential, $u_{F M}(r)=$ $4 \varepsilon\left[\left(R_{\text {int }} / r\right)^{12}-\left(R_{\text {int }} / r\right)^{6}+1 / 4\right]$ for $r<2^{1 / 6} R_{\text {int }}$ and $u_{F M}(r)=0$ otherwise. Here, we have set the energy parameter to $\varepsilon=0.1 \varepsilon_{M}$ and the interaction range to $R_{\text {int }}=0.5 \sigma_{M}$, where $\varepsilon_{M}=1.0$ and $\sigma_{M}=1.0$ correspond to the energy parameter and the diameter of the obstacle 
particles, respectively. For the comparison between the simulation and experiment, exactly the same configurations of obstacles as in the experiment are implemented. Otherwise, we use 100 statistically independent matrix structures at each density $n^{\star}=\left(N / L^{2}\right) R_{\text {int }}^{2}$, with $N$ the number of matrix particles and $L$ the linear dimension of the simulation square.

Newton's equations of motion are integrated using the velocity-Verlet algorithm [35] with a time step of $10^{-3} t_{0}$ with $t_{0}:=\left[m\left(\sigma_{M}\right)^{2} / \varepsilon_{M}\right]^{1 / 2}$ and $m=1.0$ the mass of a fluid particle. The particles carry a charge $e=1$ and a mass $m=1$ and are subjected to a uniform magnetic field $B$ that acts perpendicular to the plane of motion. The velocity of the fluid particles is fixed to a constant magnitude $v_{F}=\sqrt{2}$, which corresponds to the Fermi velocity of the 2DEG in the experiment, i.e., $v_{F}=\hbar \sqrt{2 \pi n_{e}} / m=$ $2.17 \times 10^{5} \mathrm{~m} / \mathrm{s}$, where $m$ denotes the effective electron mass in GaAs. It is associated with a cyclotron radius of $R_{\text {cy }}=m v_{F} /(e B)$ or $R_{\text {cy }}=R_{\text {int }} / \tilde{B}$, with $\tilde{B}$ being the dimensionless magnetic field $\tilde{B}=B / B_{0}$ (with $B_{0}=$ $\left.\left(m v_{F} / e R_{\text {int }}\right)\right)$. Between 100 and 2400 fluid particles per host structure are used for runs of up to $10^{6} t_{0}$. For the calculation of time averages, ten time origins per run are used, spaced equidistantly over the whole simulation time.

The conversion of units between the simulation and experiment is as follows: $\sigma_{M}=10^{-6} \mathrm{~m}$ (obstacle diameter), $m=6.097 \times 10^{-32} \mathrm{~kg}, t_{0}=9.226 \times 10^{-12} \mathrm{~s}, e=$ $1.6 \times 10^{-19} \mathrm{C}$ (electron charge), and $B_{0}=0.168 \mathrm{~T}$.

For a system of noninteracting charged particles, the conductivity $\sigma_{x x}$ is directly related to the self-diffusion constant $D$ via $\sigma_{x x}=\left(n_{e} e^{2} / m\right) D$. Hence, we can directly compare the experimentally obtained conductivity, normalized to its value at $\tilde{B}=0, \sigma_{x x}(\tilde{B}) / \sigma_{x x}(0)$, to the corresponding ratio of diffusion constants from the MD simulation, $D(\tilde{B}) / D(0)$. In the simulation, the self-diffusion constant can be obtained from the long-time limit of the mean-squared displacement (MSD) of a tagged particle, $\delta r^{2}(t)$, using the Einstein relation $D=\lim _{t \rightarrow \infty} \delta r^{2}(t) / 4 t$. Here, the MSD is defined as $\delta r^{2}(t)=\left\langle[\vec{r}(t)-\vec{r}(0)]^{2}\right\rangle$, with $\vec{r}(t)$ the position of the particle at time $t$ and $\langle\ldots\rangle$ an ensemble average.

The comparison of $\sigma_{x x}(\tilde{B}) / \sigma_{x x}(0)$ from the experiment with $D(\tilde{B}) / D(0)$ from the simulation is shown in Fig. 2(a) for different number densities. Close to the $\sigma_{x x}$ maximum, the simulation and experiment are in good agreement except for the highest $n^{\star}$. Thus, both weak localization corrections [37] and interaction effects [38] can be excluded as possible origins. The experimental values are significantly larger than the ones from the simulation at high $\tilde{B}$ fields. We tentatively attribute these deviations to a combination of quantum effects like the onset of Shubnikov-de Haas oscillations and depinning of electrons from the obstacle clusters by the residual random disorder
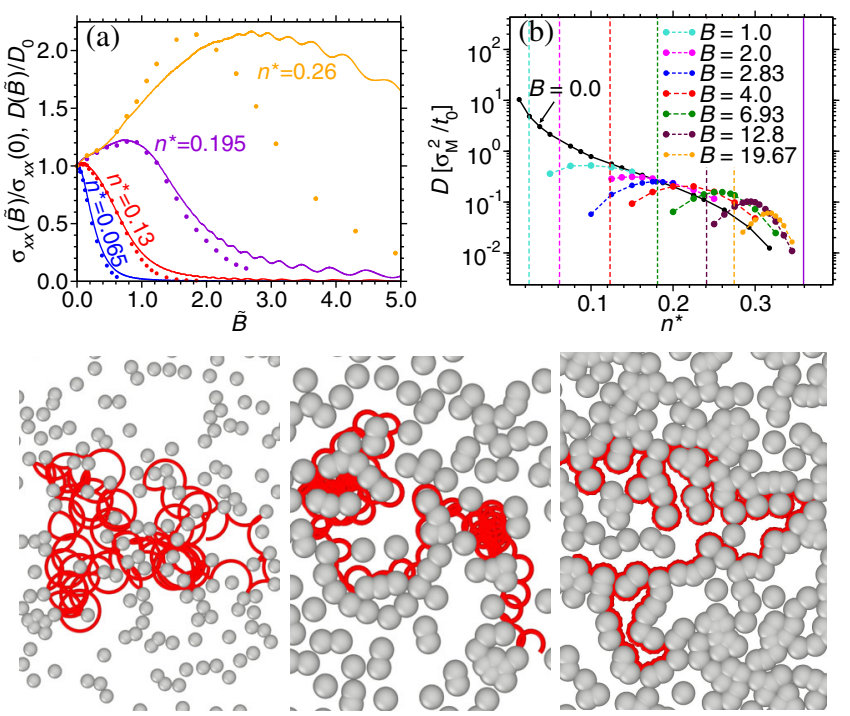

FIG. 2. (a) $\sigma_{x x}(\tilde{B}) / \sigma_{x x}(0)$ from the experiment (full lines) in comparison to $D(\tilde{B}) / D(0)$ from the simulation (full circles) for different values of $n^{\star}$. (b) $D\left(n^{\star} / n_{c}^{\star}\right)$ from the simulation for different values of $\tilde{B}$. The vertical lines correspond to the locations of $n_{c}^{\star}$ (solid line) and $n_{\mathrm{ld}, c}^{\star}(\tilde{B})$ (dashed lines). The snapshots correspond to the location of the maxima in $D\left(n^{\star}\right)$ for $\tilde{B}=0.35,1.0$, and 6.95 (from left to right). The trajectories are illustrated using OVITO [36].

[13]. However, different from the monotonic decay of $\sigma_{x x}(\tilde{B}) / \sigma_{x x}(0)$ and $D(\tilde{B}) / D(0)$ at low densities, a maximum occurs at large densities (see, e.g., the results for $n^{\star}=0.195$, where the simulation and experiment are in very good agreement around the maximum).

To get further insight into the nature of this change, we plot in Fig. 2(b) the diffusion constant from the simulation as a function of the number density, $D\left(n^{\star}\right)$, for different values of $\tilde{B}$, including $\tilde{B}=0$. At a given finite value of $\tilde{B}$, the diffusion coefficients vanish at the critical densities $n_{c}^{\star}$ and $n_{\mathrm{ld}, c}^{\star}(\tilde{B})<n_{c}^{\star}$. Therefore, at a given value of $\tilde{B}$, the function $D\left(n^{\star}\right)$ exhibits a maximum in the interval $\left[n_{\mathrm{ld}, c}^{\star}(B), n_{c}^{\star}\right]$. The snapshots in Fig. 2 show typical trajectories for different densities corresponding to the maxima in $D\left(n^{\star}\right)$ at $\tilde{B}=0.35,1.0$, and 6.95 from left to right (cf. corresponding movies in Supplemental Material [29]). These snapshots indicate a qualitative change of the motion around $\tilde{B}=1$, from a diffuse scatter of the tracer particle by the obstacle for $\tilde{B} \ll 1$ to a directed motion along the contour of the obstacle network for $\tilde{B} \gg 1$. The trajectory at $\tilde{B}=1.41$ indicates a mixture of diffuse scattering and directed motion.

The phase diagram in Fig. 3 shows $\tilde{B}$ vs $n^{\star} / n_{c}^{\star}$, with the two lines of critical points at low and high density. While the critical points at high density are independent of $\tilde{B}$ at $n^{\star} / n_{c}^{\star}=1$, the low-density critical points are located at $\tilde{B}_{\mathrm{ld}, c}=\left(\sqrt{n_{c}^{\star} / n^{\star}}-1\right)^{-1}$ [24]. The dashed red line in 


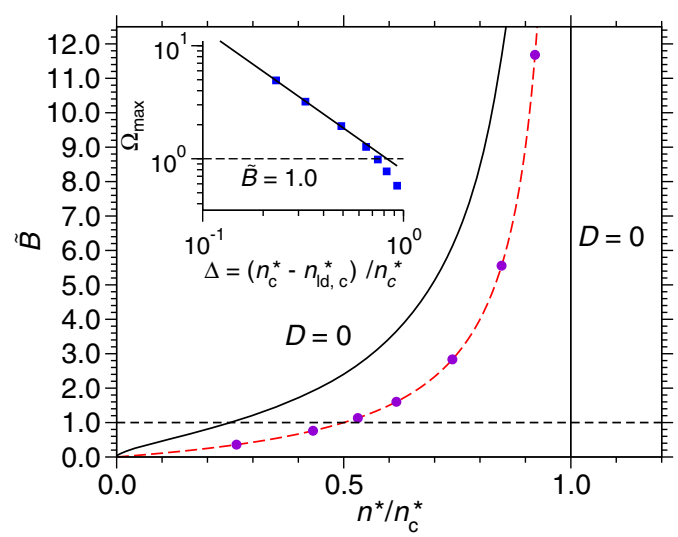

FIG. 3. Phase diagram in terms of $\tilde{B}$ vs $n^{\star} / n_{c}^{\star}$. The red dashed line is obtained from Eq. (1), and filled blue circles show $\tilde{B}_{\max }$, as determined from the simulation [cf. Fig. 2(b)]. The inset shows $\Omega_{\max }$ as a function of $\Delta$, with the solid line being a fit with $\Omega_{\max } \propto \Delta^{-1.25}$ (see the text).

between the two critical lines in Fig. 3 corresponds to the points of maximal diffusion. Its form can be understood as follows: The density $n_{\max }(\tilde{B})$ at which the diffusion coefficient is maximal is associated with two limiting cases. The maximum vanishes towards $\tilde{B} \rightarrow 0$, i.e., $n_{\max }(\tilde{B} \rightarrow 0)=0$, and it should coincide with $n_{c}^{\star}$ in the limit $\tilde{B} \rightarrow \infty$ (then $R_{\mathrm{cy}}=0$ and $n_{c}^{\star}=n_{\mathrm{ld}, c}^{\star}$ ). A function that interpolates between these two limiting cases is $n_{\max }^{\star}=$ $n_{c}^{\star} R_{\text {int }}\left(R_{\text {int }}+R_{\text {cy }}\right)^{-1}$. When this expression is solved for $\tilde{B}$, one obtains the following law for the density dependence of the reduced magnetic field at maximal diffusion:

$$
\tilde{B}_{\max }=\left(n_{c}^{\star} / n^{\star}-1\right)^{-1} .
$$

The points that are on the maximal diffusion curve in Fig. 3 are directly obtained from the data in Fig. 2(b), confirming that Eq. (1) indeed holds. Thus, remarkably, $n^{\star}\left(\tilde{B}_{\max }\right)=$ $\sqrt{n_{\mathrm{ld}, c}^{\star} n_{c}^{\star}}$. Along this line of maxima, the transport of the tracer particle changes around $\tilde{B}=1$. This can be inferred from the inset in Fig. 3, where the ratio of the diffusion constant at the maximum to that at $\tilde{B}=0$ at the corresponding density, $\Omega_{\max }=D_{\max }(B) / D\left(n_{\max }^{\star}, \tilde{B}=0\right)$, is plotted as a function of the distance between the two critical lines at a given value of $\tilde{B}, \Delta=\left(n_{c}^{\star}-n_{\mathrm{ld}, c}^{\star}\right) / n_{c}^{\star}$. For $\Delta \lesssim 0.7$, the ratio $\Omega_{\max }$ is larger than 1.0 (corresponding also to $\tilde{B}>1.0$ ), and the data can be fitted with a power law, $\Omega_{\max } \propto \Delta^{-1.25}$, indicating a divergence of this ratio towards $\tilde{B} \rightarrow \infty$. As a consequence, one expects at least strong corrections to the asymptotic critical behavior for large $\tilde{B}$ fields, and, in the limit $\tilde{B} \rightarrow \infty$, where the two critical points meet, the diffusion constant does not vanish but becomes infinite.

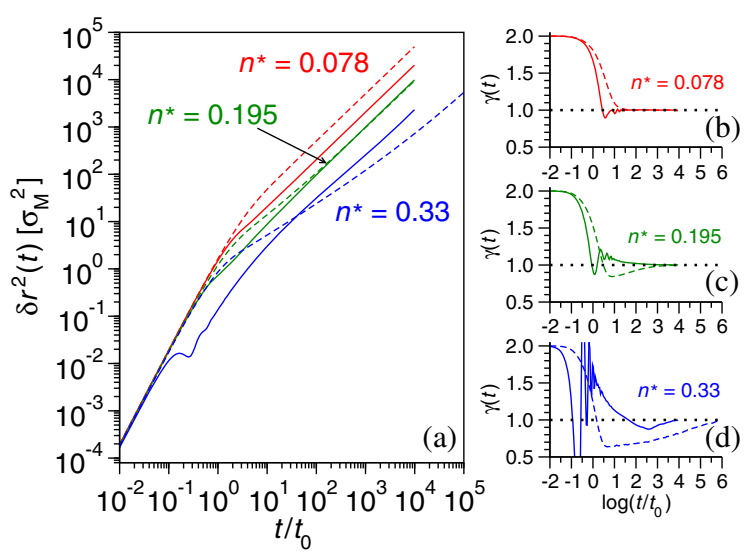

FIG. 4. (a) MSDs, $\delta r^{2}(t)$, for the densities $n^{\star}=0.078,0.195$, and 0.33 . The dashed lines are for $\tilde{B}=0$, while the solid lines correspond to the location of maximal diffusion at $\tilde{B}=0.36$, 1.02, and 7.09, respectively. Panels (b)-(d) show the local exponents $\gamma(t)$, corresponding to the MSDs in (a).

On a microscopic scale, the qualitative change of the tracer particle motion around $\tilde{B}=1$ can be analyzed in terms of MSDs. Figure 4(a) displays MSDs at three different states of maximal diffusion, $\left(n_{\max }^{\star}, \tilde{B}\right)$ (cf. the snapshots in Fig. 2 at the same states). Also included are MSDs for $\tilde{B}=0$ (dashed lines) at the corresponding densities. The onset of a directed motion along the contour of the obstacles is associated with a superlinear regime in the MSD at intermediate times for $t \gtrsim 3 t_{0}$. This is especially evident from the behavior of the local exponent of the MSD, $\gamma(t)=d \log \left[\delta r^{2}(t)\right] / d \log (t)$, which is shown in Figs. 2(b)-2(d) for the three different densities. At $n^{\star}=$ 0.078 the diffusive regime is already reached around $t=10 t_{0}$, and the diffusion for $\tilde{B}=0.36$ is slightly slower than for $\tilde{B}=0$ due to the existence of the low-density critical point in the former case. At $n^{\star}=0.195$, there is a superlinear regime for $1.0 t_{0} \lesssim t \lesssim 100 t_{0}$ at $\tilde{B}=1.02$, while in the case of $\tilde{B}=0$, a sublinear regime is seen in the same time range. A similar effect, albeit much more pronounced, is present for $n^{\star}=0.33$. Here, the $\tilde{B}=0$ curve shows an extended sublinear regime over about $2-3$ orders of magnitude due to the vicinity of the critical density $n_{c}^{\star}$. This regime is almost suppressed for $\tilde{B}=7.09$; instead, a pronounced superlinear regime and a faster transition towards normal diffusion are observed. This indicates that particularly in a dense matrix the application of a magnetic field $\tilde{B} \gg 1.0$ leads to a very efficient exploration of the matrix due to the directed motion along the contour of the obstacle matrix.

In summary, we have studied the magnetotransport through 2D disordered Lorentz gases in the classical regime. Our focus was on the nonmonotonic behavior of the conductivity and diffusion which is seen for magnetic fields $\tilde{B} \gtrsim 1.0$. We emphasize that the system under study is 
also related to the active motion of microswimmers [7-10] and thus has a more general relevance. The threshold $\tilde{B} \approx$ 1.0 marks the point where the cyclotron radius becomes smaller than the obstacle radius. This leads to the change in the motion of the tracer particle from a diffuse scatter by the obstacles to a directed motion along the obstacle contour. The latter directed motion is associated with an intermediate superlinear regime in the MSD that becomes more pronounced with increasing $\tilde{B}$.

We have shown that one can draw a line of maximal diffusion into the phase diagram that follows the law given by Eq. (1). Along this line, the diffusion constant ratio $\Omega_{\max }$ (see above) diverges in the limit $\tilde{B} \rightarrow \infty$. Thus, in this limit the directed motion dominates the transport and leads to a divergence instead of a vanishing of the diffusion coefficient. For finite $\tilde{B}$, at least strong scaling corrections are expected in the vicinity of the two critical points.

The findings presented in this Letter open the door to further experimental studies in combination with quantitative comparisons to the theory and simulation, considering different nonspherical obstacle shapes, quantum corrections, or the role of additional Gaussian disorder [13].

We thank Thomas Franosch and Herbert Spohn for useful discussions. The authors acknowledge financial support by the German Deutsche Forschungsgemeinschaft, FOR 1394 (Grant No. HO 2231/7-2). Computer time at the ZIM of the University of Düsseldorf is also gratefully acknowledged.

*horbach@thphy.uni-duesseldorf.de

†Thomas.Heinzel@hhu.de

*Present address: Laboratory for Micro- and Nanotechnology, Paul Scherrer Institute, 5232 Villigen-PSI, Switzerland.

[1] H. Lorentz, Proc. Acad. Sci. Amsterdam 7, 438 (1905).

[2] F. Höfling, T. Franosch, and E. Frey, Phys. Rev. Lett. 96, 165901 (2006).

[3] M. R. Horton, F. Höfling, J. O. Rädler, and T. Franosch, Soft Matter 6, 2648 (2010).

[4] F. Höfling and T. Franosch, Rep. Prog. Phys. 76, 046602 (2013).

[5] T. O. E. Skinner, S. K. Schnyder, D. G. A. L. Aarts, J. Horbach, and R. P. A. Dullens, Phys. Rev. Lett. 111, 128301 (2013).

[6] S. K. Schnyder, M. Spanner, F. Höfling, T. Franosch, and J. Horbach, Soft Matter 11, 701 (2015).

[7] F. Kümmel, B. ten Hagen, R. Wittkowski, I. Buttinoni, R. Eichhorn, G. Volpe, H. Löwen, and C. Bechinger, Phys. Rev. Lett. 110, 198302 (2013).

[8] E. Lauga, W. R. Diluzio, G. M. Whitesides, and H. A. Stone, Biophys. J. 90, 400 (2006).

[9] M. Han, J. Yan, S. Granick, and E. Luijten, Proc. Natl. Acad. Sci. U.S.A. 114, 7513 (2017).

[10] M. Zeitz, K. Wolff, and H. Stark, Eur. Phys. J. E 40, 23 (2017).
[11] Y. M. Beltukov and M. I. Dyakonov, Phys. Rev. Lett. 116, 176801 (2016).

[12] B. L. Altshuler, D. E. Khmelnitskii, A. I. Larkin, and P. A. Lee, Phys. Rev. B 22, 5142 (1980).

[13] A. D. Mirlin, D. G. Polyakov, F. Evers, and P. Wölfle, Phys. Rev. Lett. 87, 126805 (2001).

[14] D. G. Polyakov, F. Evers, A. D. Mirlin, and P. Wölfle, Phys. Rev. B 64, 205306 (2001).

[15] A. Dmitriev, M. Dyakonov, and R. Jullien, Phys. Rev. Lett. 89, 266804 (2002).

[16] A. Dmitriev, A. D. Mirlin, D. G. Polyakov, and M. A. Zudov, Rev. Mod. Phys. 84, 1709 (2012).

[17] L. Bockhorn, I. V. Gornyi, D. Schuh, C. Reichl, W. Wegscheider, and R. J. Haug, Phys. Rev. B 90, 165434 (2014).

[18] G. M. Gusev, P. Basmaji, Z. D. Kvon, L. V. Litvin, Y. Nastaushev, and A. I. Toropov, J. Phys. Condens. Matter 6, 73 (1994).

[19] K. Tsukagoshi, S. Wakayama, K. Oto, S. Takaoka, K. Murase, and K. Gamo, Phys. Rev. B 52, 8344 (1995).

[20] G. Lütjering, Ph.D. thesis, University of Stuttgart, 1996.

[21] G. Nachtwei, G. Lütjering, D. Weiss, Z. H. Liu, K. von Klitzing, and C. T. Foxon, Phys. Rev. B 55, 6731 (1997).

[22] G. Nachtwei, Z. H. Liu, G. Lütjering, R. R. Gerhardts, D. Weiss, K. von Klitzing, and K. Eberl, Phys. Rev. B 57, 9937 (1998).

[23] O. Yevtuchenko, G. Lütjering, D. Weiss, and K. Richter, Phys. Rev. Lett. 84, 542 (2000).

[24] A. Kuzmany and H. Spohn, Phys. Rev. E 57, 5544 (1998).

[25] W. Schirmacher, B. Fuchs, F. Höfling, and T. Franosch, Phys. Rev. Lett. 115, 240602 (2015).

[26] Y. Iye, M. Ueki, A. Endo, and S. Katsumoto, J. Phys. Soc. Jpn. 73, 3370 (2004).

[27] J. Schluck, S. Fasbender, T. Heinzel, K. Pierz, H. W. Schumacher, D. Kazazis, and U. Gennser, Phys. Rev. B 91, 195303 (2015).

[28] T. Ando, A. B. Fowler, and F. Stern, Rev. Mod. Phys. 54, 437 (1982).

[29] See Supplemental Material at http://link.aps.org/ supplemental/10.1103/PhysRevLett.120.056601 for additional experimental and simulation data as well as simulation movies.

[30] A. V. Bobylev, F. A. Maaø, A. Hansen, and E. H. Hauge, Phys. Rev. Lett. 75, 197 (1995).

[31] D. Weiss, M. L. Roukes, A. Menschig, P. Grambow, K. von Klitzing, and G. Weimann, Phys. Rev. Lett. 66, 2790 (1991).

[32] K. Ensslin and P. M. Petroff, Phys. Rev. B 41, 12307 (1990).

[33] R. Fleischmann, T. Geisel, and R. Ketzmerick, Phys. Rev. Lett. 68, 1367 (1992).

[34] S. Plimpton, J. Comput. Phys. 117, 1 (1995).

[35] K. Binder, J. Horbach, W. Kob, W. Paul, and F. Varnik, J. Phys. Condens. Matter 16, S429 (2004).

[36] A. Stukowski, Model. Simul. Mater. Sci. Eng. 18, 015012 (2010).

[37] B. L. Altshuler, A. G. Aronov, and D. E. Khmelnitsky, J. Phys. C 15, 7367 (1982).

[38] P. S. Alekseev, Phys. Rev. Lett. 117, 166601 (2016). 